\title{
La temperatura como factor de variabilidad en el desarrollo y morfología de larvas zoeas de Acanthocyclus hassleri Rathbun (Decapoda, Brachyura, Atelecyclidae) cultivadas en laboratorio
}

\author{
Erwin M. Barría ${ }^{1}$, Carlos G. Jara ${ }^{1} \&$ Kurt A. Paschke ${ }^{2}$ \\ ${ }^{1}$ Instituto de Zoología, Universidad Austral de Chile, Casilla 567, Valdivia, Chile \\ ${ }^{2}$ Instituto de Acuicultura, Universidad Austral de Chile, Casilla 1327, Puerto Montt, Chile
}

\begin{abstract}
RESUMEN. Se cultivaron larvas zoeas de Acanthocyclus hassleri Rathbun a 10, 17 y $20^{\circ} \mathrm{C}$, evaluando la sobrevivencia, tiempo de desarrollo, tamaño larval, tamaño de diferentes estructuras larvales y proporción morfométrica de esas estructuras respecto del tamaño larval. El desarrollo larval de A. hassleri comprende cuatro estadios zoea (ZI-ZIV) y una megalopa. La mayor sobrevivencia promedio fue de $95,2 \%$ a $20^{\circ} \mathrm{C}$ en ZIV, cayendo drásticamente a un $13,2 \%$ en la megalopa. El tiempo de desarrollo osciló entre $80,2 \pm 3,2$ y $28,2 \pm 0,9$ días a 10 y $20^{\circ} \mathrm{C}$ respectivamente. La ZI a $20^{\circ} \mathrm{C}$ y ZII, ZIII y ZIV a $17^{\circ} \mathrm{C}$ alcanzaron las mayores tallas larvales. El incremento de tamaño de la espina rostral, cefalotórax, abdomen y telson fue directamente proporcional a la temperatura de cultivo; mientras que para la espina dorsal, antena y ambos maxilípedos esos incrementos fueron inversamente proporcionales a la temperatura de cultivo. Los valores de proporción morfométrica de la espina rostral y abdomen permanecieron constantes; para el cefalotórax y telson disminuyó en los estadios ZIII y ZIV; mientras que para la espina rostral, espina dorsal, antena y ambos maxilípedos los tamaños relativos fueron significativamente mayores a $10^{\circ} \mathrm{C}$ que a 17 y $20^{\circ} \mathrm{C}$, a partir del estadio ZII. Los resultados de este estudio sugieren una alta capacidad de resistencia a la temperatura de las larvas zoeas de $A$. hassleri, no así de las megalopas. Tal capacidad por parte de las zoeas, se relaciona con un alto nivel de plasticidad morfo-adaptativa a la temperatura ambiental, planteando un modelo de adecuación morfológica gradual asociada con la temperatura de cultivo.
\end{abstract}

Palabras clave: Acanthocyclus hassleri, temperatura, larvas zoeas, morfometría larval, variabilidad morfológica.

\section{Temperature as factor in variability of the development and morphology of zoeae larvae of Acanthocyclus hassleri Rathbun (Decapoda, Brachyura, Atelecyclidae) reared in the laboratory}

\begin{abstract}
Zoeae larvae of A. hassleri were reared at 10,17 and $20^{\circ} \mathrm{C}$, evaluating their survival, duration of larval life, larval size, sizes of different larval structures and morphometric proportions among structures with respect to larval size. Larval development of $A$. hassleri included four zoeal stages (ZI-ZIV) and one megalopa. The highest mean zoeal survival rate was $95.2 \%$ at $20^{\circ} \mathrm{C}$ in $\mathrm{ZIV}$, decreasing to $13.2 \%$ in megalopae. Time of zoeal developmment fluctuated between $80.2 \pm 3.2 \mathrm{~d}$ at $10^{\circ} \mathrm{C}$ and $28.2 \pm 0.9 \mathrm{~d}$ at $20^{\circ} \mathrm{C}$. The largest larval sizes were reached by $\mathrm{ZI}$ at $20^{\circ} \mathrm{C}$, and ZII-IV at $17^{\circ} \mathrm{C}$. Growth in size of the rostral spine, cephalothorax, abdomen and telson were directly proportional to culture temperature; growth of the dorsal spine, antenna, and both maxillipeds were inversely proportional to culture temperature. Morphometric proportion values between the rostral spine and abdomen remained constant; for cephalothorax and telson, the proportion disminished in stages ZIII and ZIV; those of the rostral spine, dorsal spine, antenna and both maxillipeds were significately higher at $10^{\circ} \mathrm{C}$ than at 17 and $20^{\circ} \mathrm{C}$ from the $\mathrm{ZII}$ stage onward. Results suggest a high capacity for thermal resistence in the zoeae of A. hassleri but not in its' megalopae. This capacity of the zoeae represents a high level of morpho-adaptive plasticity to temperature, suggesting a model of morphological plasticity associated with the culture temperature.
\end{abstract}

Keywords: Acanthocyclus hassleri, larval culture, temperature, larval morphometry, morphological variability. 


\section{INTRODUCCIÓN}

La capacidad de dispersión de las larvas planctónicas juega un importante rol ecológico, influyendo directamente en el mantenimiento de los sistemas tróficos pelágicos y en los modelos de distribución y abundancia de las futuras poblaciones adultas (Morgan, 1990, 1992; Gaines \& Lafferty, 1995).

Específicamente para los cangrejos braquiuros, el periodo comprendido entre el inicio de la etapa postembrionaria hasta la metamorfosis está constituido por tres tipos de larvas, i.e. prezoea, zoea y megalopa (Williamson, 1982). La larva zoea tiene especial importancia en los sistemas planctónicos ya que es responsable del destino ambiental final del adulto, como consecuencia directa de su capacidad dispersiva utilizando las corrientes marinas como principal mecanismo de transporte y en menor grado por su activa capacidad de natación (Epifanio, 1987; Clancy \& Cobb, 1997; Garrison, 1999; Natunewicz \& Epifanio, 2001; Natunewicz et al., 2001).

Existe una amplia gama de factores ambientales que pueden intervenir en el desarrollo larval de los crustáceos decápodos (Villamar \& Brusca, 1988). Sin embargo, algunos autores consideran la temperatura como la principal variable abiótica que afecta las tasas de desarrollo embrionario y larval tanto en crustáceos como en el resto de los organismos poiquilotermos (Kinne, 1970; Ismael et al., 1997).

En crustáceos decápodos se ha evaluado el efecto de diferentes regímenes térmicos sobre la sobrevivencia y el tiempo de desarrollo larval (Anger, 1983; Minagawa, 1990; Sulkin \& McKeen, 1996; Anger et al., 2003); así como también sobre aspectos fisiológicos y metabolismo larval (Johns, 1981; Laughlin \& Neef; 1981; Anger et al., 1981; Ismael et al., 1997). Sin embargo, existen muy pocos trabajos que evalúen la variabilidad morfológica larvaria de crustáceos decápodos, como mecanismo de respuesta adaptativa al régimen térmico. En este sentido, Shirley et al. (1987) reportaron diferencias significativas en los morfos de larvas zoeas del cangrejo Cancer magister Dana provenientes de diferentes áreas del hemisferio norte y en larvas recién eclosionadas de embriones incubados a diferentes temperaturas. Por su parte, Wehrtmann \& Albornoz (1998) y Thatje \& Bacardit (2000) reportaron sustanciales diferencias entre las larvas zoeas de Nauticaris magellanica Milne Edwards colectadas en diferentes zonas a lo largo de la costa Pacífica y del Atlántico Sur de Sudamérica, reportando de esta manera adaptaciones morfológicas producto de la temperatura ambiental, dada esta vez por la variación latitudinal de esta variable.

En Chile, un grupo importante de cangrejos decápodos lo constituye la familia Atelecyclidae compuesta por cinco géneros (Retamal, 1981), destacando el género Acanthocyclus Edwards et Lucas con tres especies A. albatrossis Rathbun, A. gayi Edwards et Lucas y A. hassleri Rathbun, cuyas poblaciones adultas son importantes componentes en la estructuración y organización de las comunidades del intermareal rocoso del litoral chileno (Navarrete \& Castilla, 1988; Castilla et al., 1989; Carrasco \& Zamorano, 2000). De las tres especies, A. hassleri presenta el rango de distribución latitudinal más amplio, extendiéndose desde isla Alacrán, Panamá $\left(c a .8^{\circ} \mathrm{N}\right)$, hasta la costa de Valdivia, Chile (ca. $40^{\circ} \mathrm{S}$ ) (Zagal et al., 2001). Esta situación plantea la posibilidad que $A$. hassleri presenta un alto nivel de plasticidad adaptativa en sus larvas zoeas, lo que le permitiría por una parte soportar amplios rangos de temperatura, así como también ser capaces de adaptar su morfología a tales regímenes apoyando la idea planteada para las zoeas de C. magister (Shirley et al., 1987) en el hemisferio norte y para N. magellanica (Wehrtmann \& Albornoz, 1998; Thatje \& Bacardit, 2000) en el hemisferio sur. Por lo tanto, el objetivo de este trabajo es evaluar la capacidad de adaptación a la temperatura de cultivo desde el punto de vista de la sobrevivencia, tiempo de desarrollo, crecimiento, dimensiones y proporciones morfométricas de diferentes estructuras larvales de larvas zoeas de A. hassleri cultivadas en laboratorio a diferentes regímenes térmicos.

\section{MATERIALES Y MÉTODOS}

En marzo de 1999 se colectaron en forma manual 22 hembras ovígeras de A. hassleri desde el intermareal rocoso de Mehuín, Provincia de Valdivia, Chile $\left(39^{\circ} 24^{\prime} \mathrm{S}, 73^{\circ} 13^{\prime} \mathrm{W}\right)$, siendo transportadas vivas al Instituto de Zoología de la Universidad Austral de Chile en Valdivia. En el laboratorio, se seleccionaron las hembras cuyos huevos presentaron características notorias de avanzado estado de desarrollo embrionario, i.e.: presencia de embriones con ojos, abdomen claramente visible, presencia de apéndices desarrollados, escaso porcentaje de vitelo, observación de movimientos corporales internos y latidos cardíacos (Wehrtmann \& López, 2003). Se seleccionaron hembras ovígeras, 
las que fueron mantenidas individualmente en acuarios de $500 \mathrm{~mL}$ en agua de mar filtrada (filtros de 1

$\mathrm{m}$ de diámetro de poro + esterilización por radiación UV); la mantención de las hembras ovígeras se realizó bajo condiciones de temperatura y salinidad similares a las encontradas en el lugar al momento de la captura $\left(16 \pm 0,5^{\circ} \mathrm{C} ; 32 \pm 1 \mathrm{psu}\right)$ y un fotoperiodo de 12:12 h luz:oscuridad.

Aproximadamente 48 h después de la captura y selección de hembras ovígeras, tres hembras comenzaron a liberar larvas zoeas; las larvas recién eclosionadas fueron cultivadas a tres temperaturas distintas: 10,17 y $20^{\circ} \mathrm{C}$ sin aclimatación previa. Para cada uno de los tratamientos térmicos se seleccionaron larvas nacidas durante el segundo día de eclosión, dado que existía un mayor número de larvas que el primer día. Para ello se juntaron las larvas de las tres hembras en un acuario con $5 \mathrm{~L}$ de agua de mar filtrada y se seleccionaron al azar larvas zoeas con natación activa las que fueron transferidas indistintamente a dos tipos de cultivo:

a) Cultivos individuales, realizados en envases plásticos de $30 \mathrm{~mL}$. En cada tratamiento se trabajó con un número inicial de 144 larvas repartidas en tres réplicas de 48 larvas cada una. Este sistema de cultivo permitió evaluar la sobrevivencia larval, además del tiempo de desarrollo total y de cada una de las etapas de zoea.

b) Cultivos masivos, realizados en envases plásticos de $500 \mathrm{~mL}$ con una densidad inicial de 250 larvas por envase con dos réplicas por temperatura. De estos cultivos, se extrajo cada 3 a 5 días un número indeterminado de larvas de cada estadio zoea, los que fueron fijados en una solución de glicerina: formalina al 10\% en agua de mar (1:3) para su posterior análisis morfométrico.

En todos los cultivos larvales se utilizó agua de mar filtrada $(0,45 \mathrm{~m}$ de diámetro de poro) y posteriormente esterilizada con radiación $\mathrm{UV}$, realizando diariamente cambios de agua, de envase de cultivo y de suministro de alimento consistente en nauplios recién eclosionados de Artemia sp., los que fueron proporcionados ad libitum; la salinidad y el fotoperiodo se mantuvieron igual que en la mantención de las hembras ovígeras.

\section{Medición de caracteres larvales}

En larvas fijadas provenientes de cultivos masivos se midieron las siguientes estructuras: longitud total (LT) desde el ápice de la espina rostral hasta el extremo distal del telson; longitud antenal (LAn) desde la base de la escama antenal hasta el extremo distal de la antena; longitud del cefalotórax (LCe) desde la base de la escama antenal hasta el borde posterior del cefalotórax; longitud del abdomen (LAb) desde el borde anterior del primer segmento abdominal hasta el borde posterior del último segmento abdominal; longitud del telson (LTe) desde el borde de inserción con el último segmento abdominal hasta el ápice de las espinas de la furca (Fig. 1b); longitud de la espina rostral (LEr) desde el ápice de la espina rostral hasta la base del globo ocular, longitud de la espina dorsal (LEd) desde su base de fijación con el cefalotórax hasta su extremo distal, longitud del primer maxilípedo (LMI) y longitud del segundo maxilípedo (LMII) desde el punto de inserción con el cefalotórax hasta el extremo distal de las setas natatorias (Fig. 1c).

Con una lupa estereoscópica, las larvas fijadas fueron observadas y seleccionadas por estadio zoeal observando las características diagnósticas descritas por Sepúlveda (1996) para el reconocimiento de cada estadio zoea de $A$. hassleri; posteriormente se utilizó un microscopio binocular provisto de una cámara lúcida y un ocular graduado para el delineamiento del cuerpo zoeal y las estructuras larvales, posteriormente las líneas fueron escaneadas y medidas por corrección de su magnificación con el programa procesador de imágenes Scion Image para Windows que permitió transformar las unidades previamente calibradas en pixeles a unidades métricas (mm). Adicionalmente, se evaluó la proporción morfométrica de las estructuras larvales medidas como una relación entre las longitudes estructurales y la longitud total por la siguiente expresión:

Proporción morfométrica $=\frac{\text { Longitud estructural }}{\text { Longitud total }}$

De esta forma, se obtuvieron las siguientes proporciones: LEr/LT, LEd/LT, LAn/LT, LMI/LT, LMII/ LT, LCe/LT, LAb/LT y LTe/LT.

\section{Análisis estadístico}

La sobrevivencia total en cada tratamiento de cultivo y por estadios zoeales fue comparada usando un test de Chi-cuadrado $\left(\chi^{2}\right)$. Por su parte, el efecto de los diferentes regímenes térmicos sobre la duración de los estadios zoeales, longitud total de las larvas, longitudes estructurales y proporciones morfométricas para cada estadio de zoea fueron sometidos al test de normalidad de Shapiro-Wilks (Gill, 1978) y 

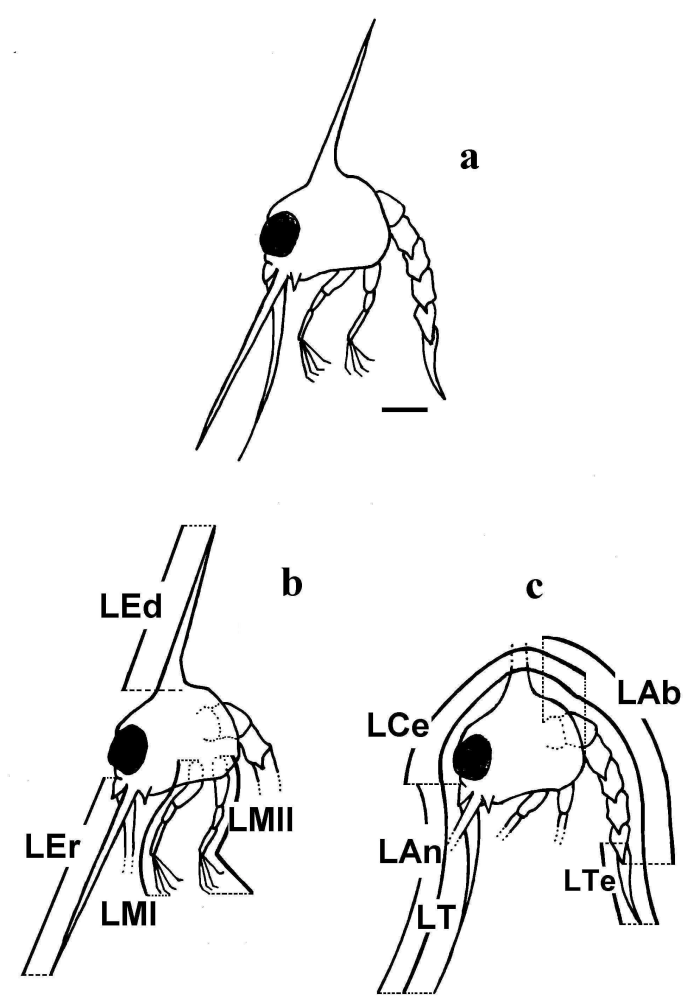

Figura 1. Estructuras larvales medidas en larvas zoeas. a) Vista lateral de una zoea I completa, b) esquema de la región anterior, c) esquema de la región anteroposterior de la larva. LT: longitud total, LAn: longitud de la antena, LCe: longitud del cefalotórax, LAb: longitud del abdomen, LTe: longitud del telson, LEd: longitud de la espina dorsal, LEr: longitud de la espina rostral LMI: longitud del primer maxilípedo, LMII: longitud del segundo maxilípedo. Escala: 0,2 mm.

Figure 1. Larval structures measuremed in zoeae. a) Lateral view of zoea I complete, b) scheme of anterior region. c) scheme of antero-posterior region. LT: total zoeal length, LAn: antennal length, LCe: cephalothoracic length, LAb: abdominal length, LTe: telson length, LEd: dorsal spine length, LEr: rostral spine length, LMI: first maxilliped length, LMII: second maxilliped length. Scale: $0.2 \mathrm{~mm}$. de homogeneidad de varianzas de Cochran. Si los datos analizados cumplieron los requerimientos para el uso de test paramétricos se utilizó un Análisis de Varianza (ANOVA) a una vía con un test a posteriori de Menor Diferencia Significativa (LSD). En caso contrario, los datos se sometieron al test de KruskalWallis de comparación de varianzas para datos no paramétricos (Sokal \& Rohlf, 1995). En el caso de las comparaciones de las proporciones morfométricas se usaron transformaciones angulares para la normalización de los datos (Sokal \& Rohlf, 1995).

\section{RESULTADOS}

\section{Sobrevivencia larval}

El desarrollo larval de A. hassleri consta de cuatro estadios zoea y una megalopa. Para efectos del experimento no se consideró las etapas postmetamórficas. La fase megalopa fue comparada sólo en términos de la sobrevivencia larval. En las tres temperaturas de cultivo, el experimento concluyó cuando todas las zoeas en estadio ZIV mudaron a megalopa.

En todos los tratamientos térmicos, tanto el porcentaje de sobrevivencia por estadio larval como la sobrevivencia acumulada fue superior al $68 \%$. El mayor porcentaje promedio de sobrevivencia acumulada se logró a los $20^{\circ} \mathrm{C}$ con un $95,1 \%$ de las larvas vivas seguido de 17 y $10^{\circ} \mathrm{C}$ con un 80,6 y $68,7 \%$ de larvas vivas, respectivamente. Sin embargo, la única diferencia significativa $(\mathrm{p}<0,05)$ se produjo debido al fuerte descenso de la sobrevivencia acumulada en la fase megalopa a $20^{\circ} \mathrm{C}$ con un porcentaje promedio de $13,2 \%$, mientras que el tratamiento a $17^{\circ} \mathrm{C}$ logró el mayor porcentaje promedio de sobrevivencia acumulada con un $77,1 \%$ seguido de $10^{\circ} \mathrm{C}$ con un $68,8 \%$ en la fase megalopa (Tabla 1 ).

\section{Duración del desarrollo zoeal}

La temperatura de cultivo tuvo un fuerte efecto tanto en el tiempo de desarrollo total como por estadios zoeales. En ambos casos, el tiempo de desarrollo disminuyó con las mayores temperaturas de cultivo (Fig. 2) presentando diferencias estadísticamente significativas $(\mathrm{p}<0,0001)$ entre los tres tratamientos de cultivo (Tabla 2). El tiempo de desarrollo zoeal completo a $20^{\circ} \mathrm{C}$ fue en promedio un $35,2 \%$ menor que a $10^{\circ} \mathrm{C}\left(80,2 \pm 3,2 \mathrm{~d}\right.$ y $28,2 \pm 0,9 \mathrm{~d}$ a 10 y $20^{\circ} \mathrm{C}$, respectivamente) (Fig. 2a). 
Tabla 1. Porcentaje de sobrevivencia para cada estadio larval y porcentaje acumulado desde la eclosión a un estadio larval dado (\% acum.) en larvas cultivadas a 10,17 y $20^{\circ} \mathrm{C} . \mathrm{n}=48$ larvas en todas las réplicas y en todas las temperaturas de cultivo.

Table 1. Percentage of survival of larval stage and cumulative percentage from hatching to a given larval stage (\% acum.) reared at 10,17 and $20^{\circ} \mathrm{C} . \mathrm{n}=48$ larvae in all replica and in all temperatures of culture.

\begin{tabular}{|c|c|c|c|c|c|c|c|c|c|c|}
\hline \multicolumn{11}{|c|}{ Temperatura } \\
\hline \multirow[t]{2}{*}{$\left({ }^{\circ} \mathrm{C}\right)$} & \multirow[t]{2}{*}{ Réplica } & \multirow{2}{*}{$\begin{array}{c}\text { Zoea I } \\
\%\end{array}$} & \multicolumn{2}{|c|}{ Zoea II } & \multicolumn{2}{|c|}{ Zoea III } & \multicolumn{2}{|c|}{ Zoea IV } & \multicolumn{2}{|c|}{ Megalopa } \\
\hline & & & $\%$ & $\%$ acum. & $\%$ & $\%$ acum. & $\%$ & $\%$ acum. & $\%$ & $\%$ acum. \\
\hline \multirow[t]{4}{*}{$10^{\circ} \mathrm{C}$} & 1 & 97,9 & 91,5 & 89,5 & 90,7 & 81,3 & 79,5 & 64,6 & 100 & 64,6 \\
\hline & 2 & 100 & 93,8 & 93,8 & 84,4 & 79,2 & 89,5 & 70,8 & 97,1 & 68,8 \\
\hline & 3 & 100 & 89,5 & 89,5 & 90,7 & 81,3 & 87,2 & 70,8 & 100 & 70,8 \\
\hline & promedio & 99,3 & 91,6 & 90,9 & 88,6 & 80,6 & 85,4 & 68,7 & 99,3 & 68,1 \\
\hline \multirow[t]{4}{*}{$17^{\circ} \mathrm{C}$} & 1 & 100 & 100 & 100 & 89,6 & 89,6 & 97,7 & 87,5 & 97,6 & 85,4 \\
\hline & 2 & 100 & 97,9 & 97,9 & 91,5 & 89,6 & 83,7 & 75,0 & 97,2 & 72,9 \\
\hline & 3 & 100 & 100 & 100 & 87,5 & 87,5 & 90,5 & 79,2 & 92,1 & 72,9 \\
\hline & promedio & 100 & 99,3 & 99,3 & 98,5 & 88,9 & 90,6 & 80,6 & 95,6 & 77,1 \\
\hline \multirow[t]{4}{*}{$20^{\circ} \mathrm{C}$} & 1 & 100 & 97,9 & 97,9 & 95,7 & 93,8 & 97,8 & 91,7 & 20,5 & 18,8 \\
\hline & 2 & 100 & 100 & 100 & 97,9 & 97,9 & 100 & 97,9 & 10,6 & 10,4 \\
\hline & 3 & 97,9 & 100 & 97,9 & 100 & 97,9 & 97,9 & 95,8 & 10,9 & 10,4 \\
\hline & promedio & 99,3 & 99,3 & 98,6 & 97,8 & 96,5 & 98,6 & 95,1 & 14,0 & 13,2 \\
\hline
\end{tabular}

Considerando el tiempo de desarrollo de cada estadio zoeal, a $10^{\circ} \mathrm{C}$ la etapa zoeal de menor duración fue el estadio ZI con 19,3 \pm 1,3 días de desarrollo, mientras que la etapa zoeal más larga correspondió al estadio ZIV con $20,8 \pm 1,8$ días de desarrollo. Las zoeas cultivadas a 17 y $20^{\circ} \mathrm{C}$, el estadio más corto fue la etapa ZII con 7,6 $\pm 0,8$ y 4,8 $\pm 1,0$ días de desarrollo para 17 y $20^{\circ} \mathrm{C}$, respectivamente. Mientras que el estadio ZIV fue el más largo con $11,6 \pm 1,3$ y $8,8 \pm 0,8$ días de desarrollo a 17 y $20^{\circ} \mathrm{C}$, respectivamente (Fig. 2b).
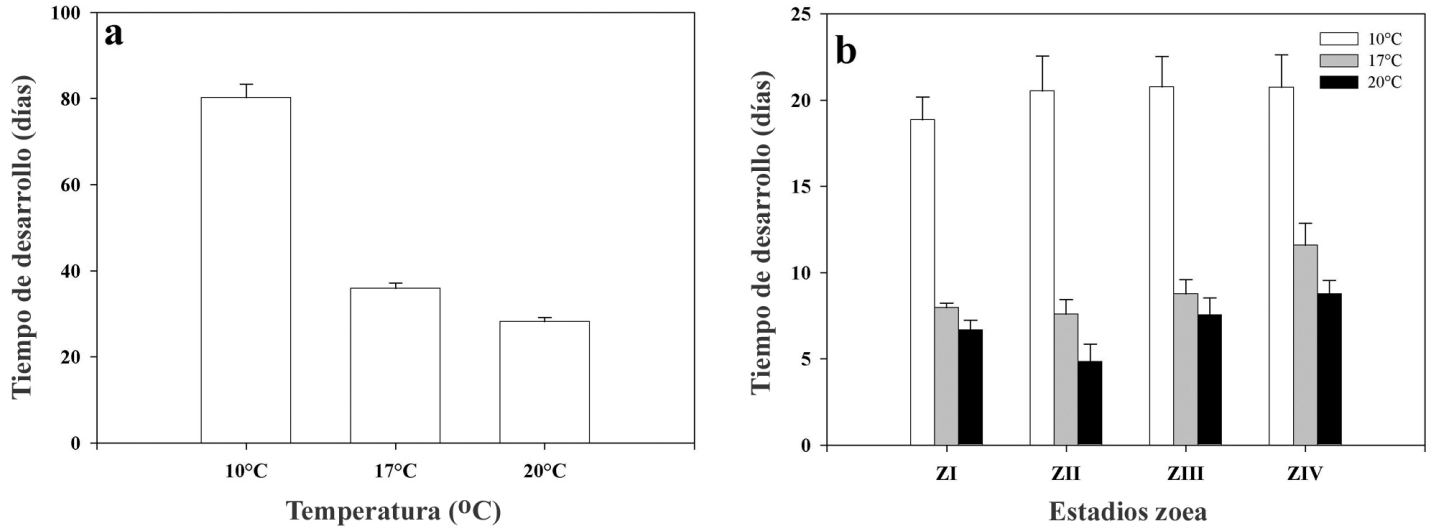

Figura 2. Tiempo de desarrollo zoeal total (a) y por estadio de zoea (b) en cultivos larvales a 10, 17 y $20^{\circ} \mathrm{C}$. Las columnas representan valores promedios ( \pm desviación estándar).

Figure 2. Time of total zoeal development (a) and by zoeal stages (b) in larval cultures at 10,17 and $20^{\circ} \mathrm{C}$. Columns represent average values ( \pm standard deviation). 
Tabla 2. Comparación estadística de la duración zoeal total y por estadios zoeales en larvas cultivadas a 10, 17 y $20^{\circ} \mathrm{C}$ (Fig. 3). gl: grados de libertad (dentro de los grupos, entre los grupos), sign: nivel de significancia, s: significativo a p $<0,0001$.

Table 2. Statistical comparisions of the total duration of zoeal development and by individual zoeal stages reared at 10,17 and $20^{\circ} \mathrm{C}$. gl: freedom degree (within groups; beetwen groups), sign: significance level, s: significative at $\mathbf{p}<0.0001$.

\begin{tabular}{|lcccccc|}
\hline \multirow{2}{*}{$\begin{array}{l}\text { Tratamientos } \\
\text { experimentales }\end{array}$} & & \multicolumn{9}{c}{ Duración de los estadios zoeales } & & \\
& & ZI & ZII & ZIII & ZIV & TOTAL \\
\hline \multirow{2}{*}{$10^{\circ} \mathrm{C}-17^{\circ} \mathrm{C}$} & valor $\boldsymbol{p}$ & 0,0000 & 0,0000 & 0,0000 & 0,0000 & 0,0000 \\
& valor F & 9138,14 & 4363,23 & 4170,23 & 1491,9 & 1770,29 \\
& gl & 1,234 & 1,223 & 1,198 & 1,176 & 1,176 \\
& sign. & $\mathrm{s}$ & $\mathrm{s}$ & $\mathrm{s}$ & $\mathrm{s}$ & $\mathrm{s}$ \\
\hline & valor $\boldsymbol{p}$ & 0,0000 & 0,0000 & 0,0000 & 0,0000 & 0,0000 \\
& valor F & 9476,11 & 6153,88 & 4948,28 & 3985,99 & 30698,35 \\
& gl & 1,234 & 1,231 & 1,212 & 1,193 & 1,193 \\
& sign. & $\mathrm{s}$ & $\mathrm{s}$ & $\mathrm{s}$ & $\mathrm{s}$ & $\mathrm{s}$ \\
\hline \multirow{2}{*}{$17^{\circ} \mathrm{C}-20^{\circ} \mathrm{C}-20^{\circ} \mathrm{C}$} & valor $\boldsymbol{p}$ & 0,0000 & 0,0000 & 0,0000 & 0,0000 & 0,0000 \\
& valor F & 619,66 & 605,80 & 111,60 & 434,42 & 3221,25 \\
& gl & 1,284 & 1,276 & 1,248 & 1,237 & 1,239 \\
& sign. & $\mathrm{s}$ & $\mathrm{s}$ & $\mathrm{s}$ & $\mathrm{s}$ & $\mathrm{s}$ \\
\hline
\end{tabular}

\section{Tamaño larval}

El tamaño larval promedio aumentó al pasar de un estadio zoea a otro en los tres tratamientos térmicos (Fig. 3). La longitud corporal de la ZI fue significativamente mayor a $20^{\circ} \mathrm{C}$ que a 10 y $17^{\circ} \mathrm{C}$; mientras que en los estadios posteriores se presentó un mayor tamaño larval a $17^{\circ} \mathrm{C}$ seguido de los tratamientos a 20 y $10^{\circ} \mathrm{C}$, respectivamente. Se presentaron diferencias de longitud larval significativamente menor ( $\mathrm{p}<0,0001$ ) en los estadios ZII y ZIV a $10^{\circ} \mathrm{C}$, mientras que en el estadio ZIII hubo diferencias en los tamaños larvales en todos los tratamientos experimentales entre sí (Tabla 3, Fig. 3).

\section{Longitud de las estructuras larvales}

En la totalidad de las estructuras medidas se observaron dos patrones de aumento de longitud a partir de la etapa ZIII: la espina rostral (LEr), cefalotórax (LCe), abdomen (LAb) y telson (LTe) registraron un aumento de longitud directamente proporcional a la temperatura de cultivo, es decir, alcanzaron mayores longitudes estructurales con las mayores temperaturas. En cambio, en la espina dorsal (LEd), antena (LAn) y ambos maxilípedos (LMI y LMII), se observó un aumento de tamaño inversamente proporcional a la temperatura de cultivo; lo que signi- fica, que crecieron más con las temperaturas de cultivo más bajas (Fig. 4).

Las variables LEr, LCe, LAb y LTe presentaron longitudes estructurales significativamente menores $(\mathrm{p}<0,05)$ en los estadios ZIII y ZIV cultivados a $10^{\circ} \mathrm{C}$. En tanto, para los dos primeros estadios (ZI y ZII), la espina rostral (LEr) presenta una longitud significativamente mayor a $20^{\circ} \mathrm{C}$ respecto de los tratamientos a 10 y $20^{\circ} \mathrm{C}$. Por su parte, la longitud cefalotoráxica (LCe) presentó una longitud significativamente mayor en la ZII cultivada a $17^{\circ} \mathrm{C}$. En el abdomen ( $\mathrm{LAb})$ en cambio, el estadio ZI presentó un tamaño estructural significativamente mayor en la larvas cultivadas a $20^{\circ} \mathrm{C}$, mientras que en el estadio ZII presentó una longitud significativamente menor en el tratamiento a $20^{\circ} \mathrm{C}$. Por último, en el telson las principales diferencias estadísticas se registraron en el estadio ZI, entre las larvas cultivadas a 10 y $17^{\circ} \mathrm{C}$ (Fig. 4, Tabla 4).

Las longitudes de la espina dorsal (LEd), antena (LAn), primer y segundo maxilípedo (LMI y LMII) presentaron valores de longitud estructural significativamente menores en los estadios ZIII y ZIV de las larvas cultivadas a $20^{\circ} \mathrm{C}$. En el estadio ZI por su parte, los valores de longitud promedio de estas cuatro estructuras fueron significativamente mayores a $20^{\circ} \mathrm{C}$ respecto del resto de los tratamien- 
Tabla 3. Análisis estadístico de la longitud corporal en larvas zoeas cultivadas a diferentes temperaturas para estadios zoeales similares. gl: grados de libertad (entre grupos, dentro de grupos), sign.: nivel de significancia, ns: no significativo, $\mathrm{s}(*)$ : significativo a $\mathrm{p}<0,05, \mathrm{~s}(* *)$ : significativo a $\mathrm{p}<0,0001$.

Table 3. Statistical analysis of the body length in zoeae larvae reared at different temperatures to similar zoeal stages. gl: freedom degree (within groups; beetwen groups), sign.: significance level, ns: non significative, $s(*)$ : significative at $\mathbf{p}<0.05, \mathrm{~s}\left({ }^{* *}\right)$ : significative at $\mathrm{p}<0.0001$.

\begin{tabular}{|c|c|c|c|c|c|}
\hline \multirow{2}{*}{$\begin{array}{c}\text { Tratamientos } \\
\text { experimentales }\end{array}$} & & \multicolumn{3}{|c|}{ Longitud total de las zoeas } & \multirow[b]{2}{*}{ ZIV } \\
\hline & & ZI & ZII & ZIII & \\
\hline \multirow[t]{4}{*}{$10^{\circ} \mathrm{C}-17^{\circ} \mathrm{C}$} & valor $\boldsymbol{p}$ & 0,7472 & 0,0000 & 0,0000 & 0,0000 \\
\hline & valor $\mathrm{F}$ & 0,10 & 43,88 & 64,81 & 179,85 \\
\hline & gl & 1,135 & 1,96 & 1,86 & 1,70 \\
\hline & sign. & ns & $\mathrm{s}(* *)$ & $\mathrm{s}(* *)$ & $\mathrm{s}(* *)$ \\
\hline \multirow[t]{4}{*}{$10^{\circ} \mathrm{C}-20^{\circ} \mathrm{C}$} & valor $\boldsymbol{p}$ & 0,0001 & 0,0000 & 0,0000 & 0,0000 \\
\hline & valor $F$ & 17,36 & 41,91 & 24,62 & 263,83 \\
\hline & $\mathrm{gl}$ & 1,147 & 1,86 & 1,70 & 1,74 \\
\hline & sign. & $\mathrm{s}(* *)$ & $\mathrm{s}(* *)$ & $\mathrm{s}(* *)$ & $\mathrm{s}(* *)$ \\
\hline \multirow[t]{4}{*}{$17^{\circ} \mathrm{C}-20^{\circ} \mathrm{C}$} & valor $\boldsymbol{p}$ & 0.0027 & 0.064 & 0.0000 & 0.5127 \\
\hline & valor $F$ & 9.37 & 3.52 & 25.35 & 0.43 \\
\hline & gl & 1.118 & 1.88 & 1.90 & 1.74 \\
\hline & sign. & $\mathrm{s}(*)$ & ns & $\mathrm{s}(* *)$ & ns \\
\hline
\end{tabular}

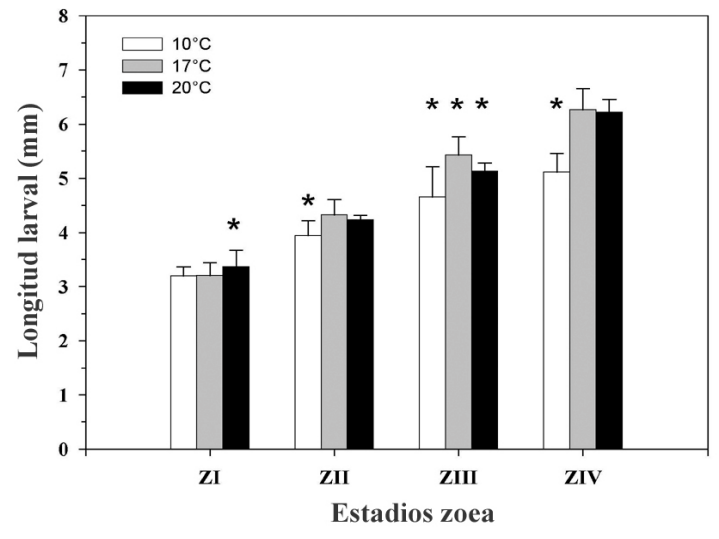

Figura 3. Longitud corporal $(\mathrm{mm})$ en larvas zoeas provenientes de cultivos a 10,17 y $20^{\circ} \mathrm{C}$. Las columnas representan valores promedios ( \pm desviación estándar). (*) diferencias estadísticamente significativas $(\mathbf{p}<0,05)$.

Figure 3. Corporal length $(\mathrm{mm})$ in zoeae larvae cultured at 10, 17 and $20^{\circ} \mathrm{C}$. Columns represent average values ( \pm standard deviation). $(*)$ statistically significative differences $(p<0.05)$. tos térmicos (Tabla 4), presentando así una respuesta opuesta a la de los estadios posteriores. El estadio ZII en cambio, conserva la tendencia de los estadios zoeales posteriores con mayores tamaños estructurales en las larvas cultivadas a $10^{\circ} \mathrm{C}$. En este estadio zoeal destaca la longitud de la espina dorsal (LEd) y del primer maxilípedo (LMI) donde los tamaños estructurales fueron significativamente mayores a $20^{\circ} \mathrm{C}$; mientras que el tamaño de la antena (LAn) es significativamente menor en las larvas cultivadas a $20^{\circ} \mathrm{C}$ y el tamaño del segundo maxilípedo es significativamente menor a $17^{\circ} \mathrm{C}$, respecto del resto de los tratamientos (Fig. 4, Tabla 4).

\section{Proporciones morfométricas de las estructuras larvales}

En general, los mayores valores de proporción morfométrica en el experimento correspondieron a la espina rostral (LEr/LT), antena (LAn/LT), espina dorsal (LEd/LT), cefalotórax (LCe/LT) y ambos maxilípedos (LMI/LT y LMII/LT); mientras que los menores valores de proporción correspondieron al abdomen (LAb/LT) y telson (LTe/LT) (Fig. 5).

Las variables LEr/LT y LAb/LT presentaron la mayor homogeneidad en los valores de proporción 
Tabla 4. Comparaciones estadísticas de longitudes estructurales entre estadios zoeales similares cultivados a diferentes temperaturas. gl: grados de libertad (entre grupos, dentro de grupos), sign.: nivel de significancia, ns: no significativo, $\left.\mathrm{s}^{*}\right)$ : significativo a $\mathrm{p}<0,05$, $\mathrm{s}(* *)$ : significativo a $\mathrm{p}<0,0001$.

Table 4. Statistic comparisions of structural length beetwen similar zoeal stages reared at different temperatures. gl: freedom degree (within groups; beetwen groups), sign.: significance level, ns: non significatives, $\mathbf{s}(*)$ : significatives at $\mathbf{p}<0.05$, $\mathbf{s}(* *)$ : significatives at $\mathbf{p}<0.0001$.

\begin{tabular}{|c|c|c|c|c|c|c|c|c|c|c|c|c|c|c|c|c|c|}
\hline \multirow{2}{*}{\multicolumn{2}{|c|}{$\begin{array}{r}\text { Tratamientos } \\
\text { experimentales }\end{array}$}} & \multicolumn{12}{|c|}{$\begin{array}{l}\text { ESTRUCTURAS LARVALES } \\
\text { LCe }\end{array}$} & \multicolumn{4}{|c|}{ LTe } \\
\hline & & ZI & ZII & ZIII & ZIV & ZI & ZII & ZIII & ZIV & ZI & ZII & ZIII & ZIV & ZI & ZII & ZIII & ZIV \\
\hline \multirow{4}{*}{$10^{\circ} \mathrm{C}-17^{\circ} \mathrm{C}$} & valor $p$ & 0,5865 & 0,0761 & 0,0000 & 0,0000 & 0,3603 & 0,0000 & 0,0000 & 0,0000 & 0,0529 & 0,0000 & 0,0000 & 0,9645 & 0,003 & 0,4567 & 0,0000 & 0,0000 \\
\hline & valor $F$ & 0,3 & 3,22 & 29,74 & 87,66 & 0,84 & 26,22 & 39,2 & 34,36 & 3,81 & 67,50 & 22,44 & 0,00 & 9,15 & 0,56 & 147,37 & 220,98 \\
\hline & $\mathrm{gl}$ & 1,135 & 1,96 & 1,86 & 1,70 & 1,135 & 1,96 & 1,86 & 1,70 & 1,135 & 1,96 & 1,86 & 1,70 & 1,135 & 1,96 & 1,86 & 1,70 \\
\hline & sign. & $\mathrm{ns}$ & ns & $\mathrm{s}(* *)$ & $\mathrm{s}(* *)$ & ns & $\mathrm{s}(* *)$ & $\mathrm{s}(* *)$ & $\mathrm{s}(* *)$ & $\mathrm{ns}$ & $\mathrm{s}(* *)$ & $\mathrm{s}(* *)$ & $\mathrm{ns}$ & $\mathrm{s}(*)$ & $\mathrm{ns}$ & $\mathrm{s}(* *)$ & $\mathrm{s}(* *)$ \\
\hline \multirow[t]{4}{*}{$10^{\circ} \mathrm{C}-20^{\circ} \mathrm{C}$} & valor $\boldsymbol{p}$ & 0,000 & 0,003 & 0,0000 & 0,8509 & 0,3271 & 0,6160 & 0,0000 & 0,0000 & 0,0001 & 0,000 & 0,0000 & 0,3690 & 0,1854 & 0,5206 & 0,0000 & 0,0000 \\
\hline & valor $\mathrm{F}$ & 28,40 & 14,10 & 17,77 & 0,04 & 0,97 & 0,25 & 21,1 & 27,46 & 15,34 & 70,83 & 21,77 & 0,82 & 1,77 & 0,42 & 68,92 & 96,88 \\
\hline & $\mathrm{gl}$ & 1,147 & 1,88 & 1,70 & 1,74 & 1,147 & 1,86 & 1,70 & 1,74 & 1,147 & 1,86 & 1,70 & 1,74 & 1,147 & 1,86 & 1,70 & 1,74 \\
\hline & sign. & $\mathrm{s}(* *)$ & $\mathrm{s}(*)$ & $\mathrm{s}(* *)$ & ns & $\mathrm{ns}$ & $\mathrm{ns}$ & $\mathrm{s}(* *)$ & $\mathrm{s}(* *)$ & $\mathrm{s}(* *)$ & $\mathrm{s}(* *)$ & $\mathrm{s}(* *)$ & $\mathrm{ns}$ & ns & $\mathrm{ns}$ & $\mathrm{s}(* *)$ & $\mathrm{s}(* *)$ \\
\hline \multirow[t]{6}{*}{$17^{\circ} \mathrm{C}-20^{\circ} \mathrm{C}$} & valor $\boldsymbol{p}$ & 0,001 & 0,008 & 0,0799 & 0,0000 & 0,9891 & 0,0000 & 0,0130 & 0,1393 & 0,1002 & 0,3479 & 0,9051 & 0,1925 & 0,0715 & 0,0636 & 0,0000 & 0,0002 \\
\hline & valor $\mathrm{F}$ & 17,15 & 12,19 & 3,14 & 95,93 & 0,00 & 31,08 & 6,42 & 2,23 & 2,75 & 0,89 & 0,01 & 1,73 & 3,31 & 3,53 & 26,83 & 15,89 \\
\hline & $\mathrm{gl}$ & 1,118 & 1,86 & 1,90 & 1,74 & 1,118 & 1,88 & 1,90 & 1,74 & 1,118 & 1,88 & 1,90 & 1,74 & 1,118 & 1,88 & 1,90 & 1,74 \\
\hline & sign. & $\mathrm{s}(* *)$ & $\mathrm{s}(*)$ & $\mathrm{ns}$ & $\mathrm{s}(* *)$ & $\mathrm{ns}$ & $\mathrm{s}(* *)$ & $\mathrm{s}(*)$ & $\mathrm{ns}$ & $\mathrm{ns}$ & $\mathrm{ns}$ & $\mathrm{ns}$ & $\mathrm{ns}$ & $\mathrm{ns}$ & $\mathrm{ns}$ & $\mathrm{s}(* *)$ & $\mathrm{s}(*)$ \\
\hline & & \multicolumn{4}{|c|}{ LEd } & \multicolumn{4}{|c|}{ LAn } & \multicolumn{4}{|c|}{ LMI } & \multicolumn{4}{|c|}{ LMII } \\
\hline & & ZI & ZII & ZIII & ZIV & ZI & ZII & ZIII & ZIV & ZI & ZII & ZIII & ZIV & ZI & ZII & ZIII & ZIV \\
\hline \multirow[t]{4}{*}{$10^{\circ} \mathrm{C}-17^{\circ} \mathrm{C}$} & valor $p$ & 0,9713 & 0,0055 & 0,8429 & 0,0007 & 0,954 & 0,1636 & 0,8980 & 0,6184 & 0,6704 & 0,0020 & 0,0957 & 0,3560 & 0,2094 & 0,0002 & 0,1193 & 0,2667 \\
\hline & valor $F$ & 0 & 8,07 & 0,04 & 12,62 & 0,00 & 1,97 & 0,02 & 0,26 & 0,18 & 10,13 & 2,84 & 0,86 & 1,59 & 14,546 & 2,48 & 1,25 \\
\hline & $\mathrm{gl}$ & 1.113 & 1,94 & 1,86 & 1,70 & 1,130 & 1,93 & 1,84 & 1,67 & 1,131 & 1,95 & 1,86 & 1,70 & 1,115 & 1,96 & 1,86 & 1,70 \\
\hline & sign. & $\mathrm{ns}$ & $\mathrm{s}(*)$ & ns & $\mathrm{s}(*)$ & $\mathrm{ns}$ & $\mathrm{ns}$ & $\mathrm{ns}$ & ns & ns & $\mathrm{s}(*)$ & $\mathrm{ns}$ & $\mathrm{ns}$ & $\mathrm{ns}$ & $\mathrm{s}(*)$ & $\mathrm{ns}$ & ns \\
\hline \multirow[t]{4}{*}{$10^{\circ} \mathrm{C}-20^{\circ} \mathrm{C}$} & valor $p$ & 0,0006 & 0,0033 & 0,0000 & 0,0000 & 0,0000 & 0,0000 & 0,0001 & 0,0009 & 0,0025 & 0,0058 & 0,0001 & 0,0002 & 0,0185 & 0,1288 & 0,0000 & 0,0001 \\
\hline & valor $\mathrm{F}$ & 12,42 & 9,16 & 37,06 & 74,03 & 17,63 & 20,34 & 18,39 & 7,21 & 9,47 & 8,02 & 16,11 & 14,88 & 5,70 & 2,35 & 27,04 & 17,52 \\
\hline & $\mathrm{gl}$ & 1.145 & 1,84 & 1,70 & 1,74 & 1,143 & 1,85 & 1,68 & 1,71 & 1,143 & 1,85 & 1,70 & 1,74 & 1,127 & 1,86 & 1,70 & 1,74 \\
\hline & sign. & $\mathrm{s}(*)$ & $\mathrm{s}(*)$ & $\mathrm{s}(* *)$ & $\mathrm{s}(* *)$ & $\mathrm{s}(* *)$ & $\mathrm{s}(* *)$ & $\mathrm{s}(* *)$ & $\mathrm{s}(*)$ & $\mathrm{s}(*)$ & $\mathrm{s}(*)$ & $\mathrm{s}(* *)$ & $\mathrm{s}(*)$ & $\mathrm{s}(*)$ & $\mathrm{ns}$ & $\mathrm{s}(* *)$ & $\mathrm{s}(* *)$ \\
\hline \multirow[t]{4}{*}{$17^{\circ} \mathrm{C}-20^{\circ} \mathrm{C}$} & valor $\boldsymbol{p}$ & 0,0051 & 0,7722 & 0,0000 & 0,0000 & 0,011 & 0,0000 & 0,0000 & 0,0000 & 0,0029 & 0,8668 & 0,0087 & 0,0000 & 0,0030 & 0,0006 & 0,0001 & 0,0007 \\
\hline & valor $\mathrm{F}$ & 8,15 & 0,08 & 60,68 & 119,8 & 11,23 & 20,57 & 20,57 & 27,74 & 9,22 & 0,03 & 7,20 & 30,96 & 9,27 & 12,63 & 15,59 & 12,60 \\
\hline & $\mathrm{gl}$ & 1.117 & 1,88 & 1,90 & 1,74 & 1,113 & 1,86 & 1,86 & 1,74 & 1,116 & 1,88 & 1,90 & 1,74 & 1,100 & 1,88 & 1,90 & 1,74 \\
\hline & sign. & $\mathrm{s}(*)$ & ns & $\mathrm{s}(* *)$ & $\mathrm{s}(* *)$ & $\mathrm{s}(*)$ & $\mathrm{s}(* *)$ & $\mathrm{s}(* *)$ & $\mathrm{s}(* *)$ & $\mathrm{s}(*)$ & $\mathrm{ns}$ & $\mathrm{s}(*)$ & $\mathrm{s}(* *)$ & $\mathrm{s}(*)$ & $\mathrm{s}(*)$ & $\mathrm{s}(* *)$ & $\mathrm{s}(*)$ \\
\hline
\end{tabular}




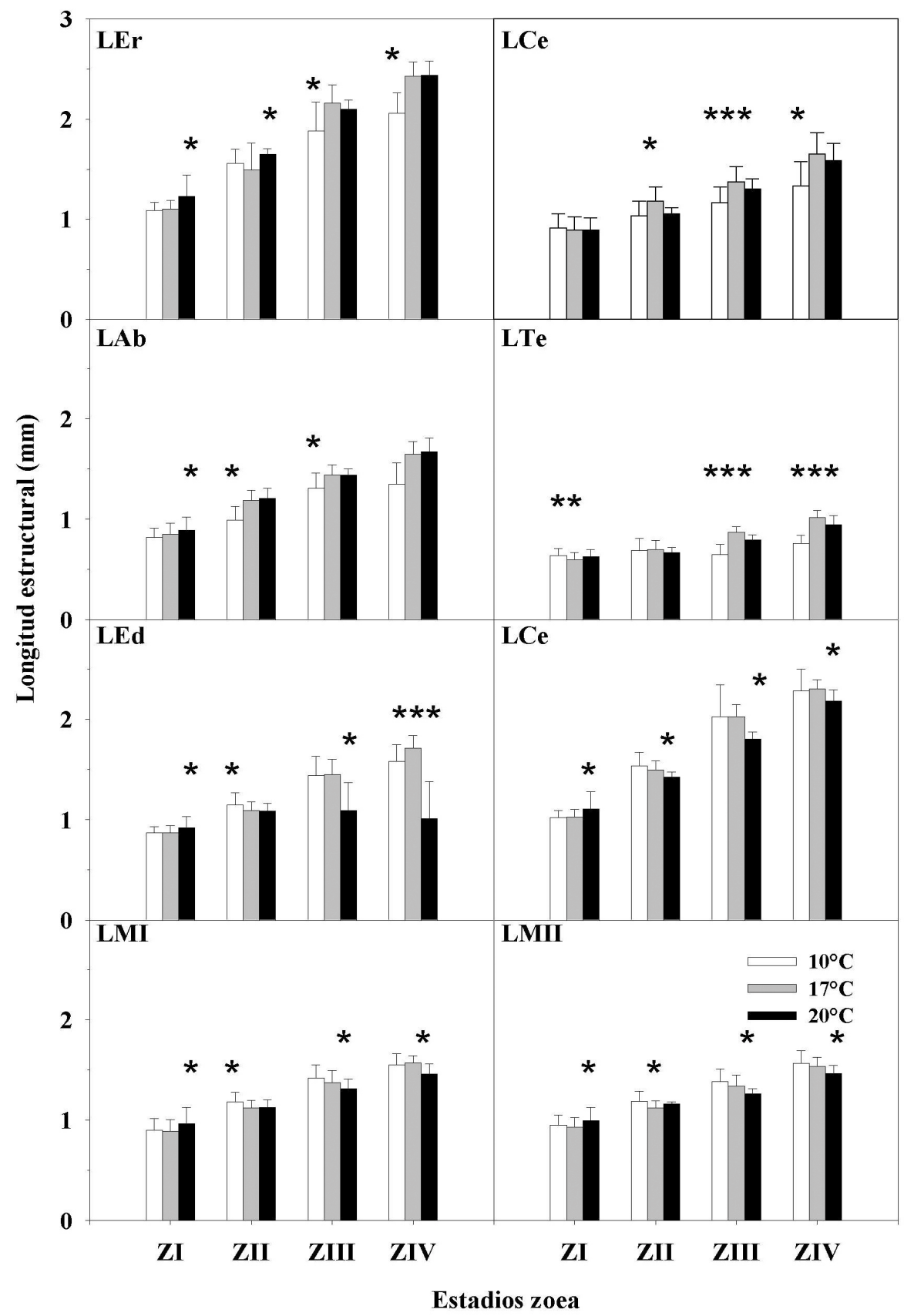

Figura 4. Longitud (mm) de espina rostral (LEr), cefalotórax (LCe), abdomen (LAb), telson (LTe), espina dorsal (LEd), antena (LAn), primer maxilípedo (LMI) y segundo maxilípedo (LMII) en larvas zoeas cultivadas a 10,17 y $20^{\circ} \mathrm{C}$. Las columnas representan valores promedios ( \pm desviación estándar). $(*)$ diferencias estadísticamente significativas $(\mathbf{p}<\mathbf{0 , 0 5})$.

Figure 4. Length (mm) of rostral spine (LEr), cephalothorax (LCe), abdomen (LAb), telson (LTe), dorsal spine (LEd), antenna (LAn), first maxilliped (LMI) and second maxilliped (LMII) in zoeae reared at 10, 17 and $20^{\circ} \mathrm{C}$. Columns represent average values $( \pm$ standard deviation). $(*)$ statistically significative differences $(p<0.05)$. 
morfométrica durante todo el desarrollo zoeal con algunos valores levemente significativos como: los estadios ZI a $20^{\circ} \mathrm{C}, \mathrm{ZII}$ a $17^{\circ} \mathrm{C}, \mathrm{ZIII}$ a 17 y $20^{\circ} \mathrm{C}$, ZIV a 10 y $17^{\circ} \mathrm{C}$ para la variable $\mathrm{LEr} / \mathrm{LT}$; o los estadios ZI a $17^{\circ} \mathrm{C}$, ZII entre las tres temperaturas de cultivo, y ZIII a $17^{\circ} \mathrm{C}$ para la variable LAb/LT. Por su parte, las variables LCe/LT y LTe/LT presentaron una tendencia similar en los estadios ZI y ZII, con mayores valores de proporción morfométrica a $17^{\circ} \mathrm{C}$ en ZI y $10^{\circ} \mathrm{C}$ en ZII; en los estadios ZIII y ZIV en cambio, los valores de proporción morfométrica fueron homogéneos para el cefalotórax (LCe/LT) en los estadios ZIII y ZIV, mientras que para el telson (LTe/LT) el valor de proporción morfométrica fue significativamente menor a $10^{\circ} \mathrm{C}$ y significativamente mayor a $17^{\circ} \mathrm{C}$ en el estadio ZIV (Fig. 5, Tabla 5).

Las proporciones morfométricas de la espina rostral (LEr/LT), espina dorsal (LEd/LT), antena (LAn/LT), primer y segundo maxilípedo (LMI/LT y LMII/LT) presentaron mayores valores de proporción en larvas cultivadas a $10^{\circ} \mathrm{C}$ a partir del estadio ZII. Las diferencias significativas más notables entre los tres tratamientos térmicos fueron apreciadas en los estadios ZII, ZIII y ZIV para las variables LEd/LT, LAn/LT, LMI/LT y LMII/LT (Fig. 5, Tabla $5)$.

\section{DISCUSIÓN}

Los altos niveles de sobrevivencia zoeal en este estudio muestran la capacidad euritérmica de las larvas zoeas de A. hassleri. Algunos autores (Sastry, 1983; Pechenick, 1987) han establecido como modelo general de comportamiento en cultivos larvales de invertebrados marinos que se encuentren dentro del rango térmico fisiológicamente compatible con el desarrollo larvario, las altas temperaturas aceleran el tiempo de desarrollo a la vez que disminuyen la sobrevivencia. En el caso de A. hassleri, este patrón es observado aunque no tan claramente hasta el estadio ZIV, donde no existen mayores diferencias en los niveles de sobrevivencia zoeal pudiendo soportar temperaturas superiores a $23^{\circ} \mathrm{C}$. Sin embargo, en la fase megalopa la sobrevivencia desciende de manera importante a $20^{\circ} \mathrm{C}$, siendo finalmente el experimento a $17^{\circ} \mathrm{C}$ el que logró el mayor porcentaje de larvas megalopas (Tabla 1). El alto nivel de resistencia a altas temperaturas por parte de las larvas zoeas se encuentra respaldado en el ambiente por el hallazgo de estadios zoea del género
Acanthocyclus en muestras de plancton colectadas en la zona norte de Chile entre Arica $\left(c a .18^{\circ} \mathrm{S}\right)$ y Puerto Huasco $\left(\mathrm{ca} .28^{\circ} \mathrm{S}\right)$ sometidas a una fuerte influencia del evento El Niño de los años 1982-83 (Báez, 1997). Por su parte, el drástico descenso de la sobrevivencia de la fase megalopa a $20^{\circ} \mathrm{C}$ plantea la idea que esta fase larval es fisiológicamente más sensible que los estadios zoeales a las altas temperaturas. Respuestas similares han sido observadas en otras especies de cangrejos braquiuros e.g. $C$. magister (Sulkin et al., 1996) y Chasmagnathus granulata Dana (Ismael et al., 1997), las que son atribuibles a bajas de la actividad metabólica reflejadas básicamente por descensos en la tasa respiratoria, tasa de crecimiento y alimentación producto de los profundos cambios en el desarrollo debido a la transición de una larva nadadora a un cangrejo bentónico caminador (Anger, 2001). Además, siendo la fase de megalopa controlada por factores asociados al hábitat de los adultos, los que se relacionan directamente con el asentamiento y metamorfosis de los individuos, tales como: características del sustrato, presencia de adultos conespecíficos o congenéricos entre otros (Gebauer et al., 2003).

Respecto a la fisiología larval, ha sido ampliamente estudiada la relación de proporcionalidad inversa que existe entre la temperatura de cultivo y el tiempo de desarrollo larval como consecuencia directa de la temperatura ambiental sobre el ritmo de actividad metabólica larval (Anger, 2001). Por lo tanto, en el presente experimento las diferencias significativas (Tabla 2) en la disminución de los tiempos de desarrollo zoeal total con las mayores temperaturas de cultivo (Fig. 3), no constituyen una excepción a lo descrito anteriormente.

Si se considera que la duración del desarrollo de las fases larvales pelágicas determina el tiempo de permanencia de las larvas en el plancton, lo que a su vez tiene una directa incidencia sobre el potencial de dispersión larval de una especie (Palumbi, 1995; Levin \& Bridges, 1995); esto permite plantear un modelo de potencialidad dispersiva en las larvas zoeas de A. hassleri proyectado en un gradiente latitudinal. Dado que esta especie se distribuye desde Panamá hasta la costa centro-sur de Chile, cubriendo aproximadamente $48^{\circ}$ de latitud en su distribución geográfica; se puede establecer que en límite norte de su rango geográfico, por la mayor temperatura del mar, el desarrollo larval es de duración más reducida y por lo tanto las larvas dispersan a corta distancia, mientras que en el límite sur de su distribución como la temperatura del agua es 
Tabla 5. Comparación estadística de proporciones morfométricas de longitud de las estructuras larvales respecto de la longitud corporal entre estadios zoeales similares cultivados a 10, 17 y $20^{\circ} \mathrm{C}$. gl: grados de libertad (entre grupos, dentro de grupos), sign.: nivel de significancia, ns: no significativo, $\mathrm{s}(*)$ : significativo a $\mathrm{p}<$ $0,05, \mathrm{~s}(* *)$ : significativo a $\mathrm{p}<0,0001$.

Table 5. Statistics comparisions of morphometrics proportions of larval structures length respect to zoeal body length beetwen similar zoeal stages reared at 10,17 and $20^{\circ} \mathrm{C}$. gl: freedom degree (within groups; beetwen groups), sign: significance level, ns: non significatives, $\mathbf{s}(*)$ : significatives at $\mathbf{p}<0.05$, $\mathbf{s}(* *)$ : significatives at $\mathbf{p}$ $<0.0001$.

\begin{tabular}{|c|c|c|c|c|c|c|c|c|c|c|c|c|c|c|c|c|c|}
\hline \multicolumn{2}{|c|}{$\begin{array}{l}\text { Tratamientos } \\
\text { experimentales }\end{array}$} & \multicolumn{4}{|c|}{ LEr/LT } & \multicolumn{5}{|c|}{$\begin{array}{l}\text { ESTRUCTURAS LARVALES } \\
\text { LCe/LT }\end{array}$} & \multicolumn{3}{|c|}{ LAb/LT } & \multicolumn{4}{|c|}{ LTe/LT } \\
\hline \multirow[t]{4}{*}{$10^{\circ} \mathrm{C}-17^{\circ} \mathrm{C}$} & valor $p$ & 0,6934 & 0,0000 & 0,2096 & 0,0192 & 0,6779 & 0,0903 & 0,4113 & 0,7595 & 0,0088 & 0,0000 & 0,0000 & 0,9465 & 0,0023 & 0,0220 & 0,0000 & 0,0002 \\
\hline & valor F & 0,16 & 39,64 & 1,26 & 5,74 & 0,17 & 2,93 & 0,68 & 0,09 & 7,07 & 18,89 & 22,27 & 0,00 & 9,63 & 5,42 & 107,64 & 14,98 \\
\hline & $\mathrm{gl}$ & 1,135 & 1,96 & 1,86 & 1,70 & 1,129 & 1,96 & 1,86 & 1,70 & 1,135 & 1,96 & 1,86 & 1,70 & 1,135 & 1,96 & 1,86 & 1,70 \\
\hline & sign. & $\mathrm{ns}$ & $\mathrm{s}(* *)$ & ns & $\mathrm{s}(*)$ & $\mathrm{ns}$ & $\mathrm{ns}$ & ns & $\mathrm{ns}$ & $\mathrm{s}(*)$ & $\mathrm{s}(* *)$ & $\mathrm{s}(* *)$ & ns & $\mathrm{s}(*)$ & $\mathrm{s}(*)$ & $\mathrm{s}(* *)$ & $\mathrm{s}(*)$ \\
\hline \multirow[t]{4}{*}{$10^{\circ} \mathrm{C}-20^{\circ} \mathrm{C}$} & valor $\boldsymbol{p}$ & 0,0000 & 0,1488 & 0,4376 & 0,096 & 0,0008 & 0,0010 & 0,2882 & 0,3924 & 0,2162 & 0,0000 & 0,5402 & 0,3690 & 0,011 & 0,0031 & 0,0000 & 0,5033 \\
\hline & valor $\mathrm{F}$ & 17,50 & 2,12 & 0,61 & 2,84 & 11,82 & 11,60 & 1,15 & 0,74 & 1,55 & 29,21 & 0,38 & 0,82 & 11,16 & 9,24 & 58,59 & 0,45 \\
\hline & $\mathrm{gl}$ & 1,147 & 1,86 & 1,70 & 1,74 & 1,141 & 1,86 & 1,70 & 1,74 & 1,118 & 1,86 & 1,70 & 1,74 & 11,47 & 1,86 & 1,70 & 1,74 \\
\hline & sign. & $\mathrm{s}(* *)$ & ns & $\mathrm{ns}$ & $\mathrm{ns}$ & $\mathrm{s}(*)$ & $\mathrm{s}(*)$ & $\mathrm{ns}$ & $\mathrm{ns}$ & ns & $\mathrm{s}(* *)$ & ns & ns & $\mathrm{s}(*)$ & $\mathrm{s}(*)$ & $\mathrm{s}(* *)$ & ns \\
\hline \multirow[t]{6}{*}{$17^{\circ} \mathrm{C}-20^{\circ} \mathrm{C}$} & valor $\boldsymbol{p}$ & 0,0015 & 0,0000 & 0,0004 & 0,3998 & 0,0026 & 0,0000 & 0,7824 & 0,1430 & 0,0595 & 0,0236 & 0,0000 & 0,1925 & 0,0000 & 0,251 & 0,0891 & 0,0004 \\
\hline & valor $\mathrm{F}$ & 10,56 & 29,17 & 13,38 & 0,74 & 9,52 & 39,18 & 0,08 & 2,19 & 3,61 & 5,31 & 30,70 & 1,73 & 18,19 & 1,34 & 2,95 & 13,79 \\
\hline & $\mathrm{gl}$ & 1,118 & 1,88 & 1,90 & 1,74 & 1,112 & 1,88 & 1,90 & 1,74 & 1,147 & 1,88 & 1,90 & 1,74 & 1,118 & 1,88 & 1,90 & 1,73 \\
\hline & sign. & $\mathrm{s}(*)$ & $\mathrm{s}(* *)$ & $\mathrm{s}(*)$ & ns & $\mathrm{s}(*)$ & $\mathrm{s}(* *)$ & ns & $\mathrm{ns}$ & $\mathrm{ns}$ & $\mathrm{s}(*)$ & $\mathrm{s}(* *)$ & $\mathrm{ns}$ & $\mathrm{s}(* *)$ & ns & ns & $\mathrm{s}(*)$ \\
\hline & & \multicolumn{4}{|c|}{ LEd/LT } & \multicolumn{4}{|c|}{ LAn/LT } & \multicolumn{4}{|c|}{ LMI/LT } & \multicolumn{4}{|c|}{ LMII/LT } \\
\hline & & ZI & ZII & ZIII & ZIV & ZI & ZII & ZIII & ZIV & ZI & ZII & ZIII & ZIV & ZI & ZII & ZIII & ZIV \\
\hline \multirow[t]{4}{*}{$10^{\circ} \mathrm{C}-17^{\circ} \mathrm{C}$} & valor $\boldsymbol{p}$ & 0,0073 & 0,0000 & 0,0000 & 0,0000 & 0,0614 & 0,0000 & 0,0000 & 0,0000 & 0,2737 & 0,0000 & 0,0000 & 0,0000 & 0,9861 & 0,0000 & 0,0000 & 0,0000 \\
\hline & valor $F$ & 7,43 & 36,96 & 95,24 & 35,40 & 3,56 & 49,93 & 171,14 & 70,75 & 1,21 & 84,61 & 106,41 & 150,45 & 0,00 & 81,71 & 63,40 & 130,09 \\
\hline & $\mathrm{gl}$ & 1,132 & 1,94 & 1,86 & 1,70 & 1,130 & 1,93 & 1,84 & 1,67 & 1,131 & 1,96 & 1,86 & 1,70 & 1,115 & 1,96 & 1,86 & 1,70 \\
\hline & sign. & $\mathrm{s}(*)$ & $\mathrm{s}(* *)$ & $\mathrm{s}(* *)$ & $\mathrm{s}(* *)$ & ns & $\mathrm{s}(* *)$ & $\mathrm{s}(* *)$ & $\mathrm{s}(* *)$ & ns & $\mathrm{s}(* *)$ & $\mathrm{s}(* *)$ & $\mathrm{s}(* *)$ & ns & $\mathrm{s}(* *)$ & $\mathrm{s}(* *)$ & $\mathrm{s}(* *)$ \\
\hline \multirow[t]{4}{*}{$10^{\circ} \mathrm{C}-20^{\circ} \mathrm{C}$} & valor $\boldsymbol{p}$ & 0,6696 & 0,0000 & 0,0000 & 0,0000 & 0,0913 & 0,0000 & 0,0000 & 0,0000 & 0,3876 & 0,0000 & 0,0000 & 0,0000 & 0,5634 & 0,0000 & 0,0000 & 0,0000 \\
\hline & valor $F$ & 0,18 & 40,53 & 94,37 & 185,71 & 2,89 & 91,46 & 247,56 & 125,45 & 0,75 & 58,53 & 76,16 & 246,79 & 0,34 & 33,84 & 55,62 & 198,17 \\
\hline & $\mathrm{gl}$ & 1,145 & 1,84 & 1,70 & 1,74 & 1,143 & 1,85 & 1,68 & 1,71 & 1,143 & 1,86 & 1,70 & 1,74 & 1,127 & 1,86 & 1,70 & 1,74 \\
\hline & sign. & $\mathrm{ns}$ & $\mathrm{s}(* *)$ & $\mathrm{s}(* *)$ & $\mathrm{s}(* *)$ & $\mathrm{ns}$ & $\mathrm{s}(* *)$ & $\mathrm{s}(* *)$ & $\mathrm{s}(* *)$ & $\mathrm{ns}$ & $\mathrm{s}(* *)$ & $\mathrm{s}(* *)$ & $\mathrm{s}(* *)$ & $\mathrm{ns}$ & $\mathrm{s}(* *)$ & $\mathrm{s}(* *)$ & $\mathrm{s}(* *)$ \\
\hline \multirow[t]{4}{*}{$17^{\circ} \mathrm{C}-20^{\circ} \mathrm{C}$} & valor $\boldsymbol{p}$ & 0,0233 & 0,6575 & 0,0000 & 0,0000 & 0,5962 & 0,0094 & 0,0000 & 0,0030 & 0,7292 & 0,1603 & 0,5297 & 0,0005 & 0,6776 & 0,0000 & 0,8326 & 0,0259 \\
\hline & valor $F$ & 5,29 & 0,2 & 42,23 & 110,86 & 0,28 & 7,05 & 50,56 & 9,39 & 0,12 & 2,01 & 0,40 & 13,44 & 0,17 & 33,84 & 0,04 & 5,17 \\
\hline & $\mathrm{gl}$ & 1,115 & 1,88 & 1,90 & 1,74 & 1,113 & 1,86 & 1,90 & 1,74 & 1,116 & 1,88 & 1,90 & 1,74 & 1,100 & 1,88 & 1,89 & 1,74 \\
\hline & sign. & $\mathrm{s}(*)$ & ns & $\mathrm{s}(* *)$ & $\mathrm{s}(* *)$ & $\mathrm{ns}$ & $\mathrm{s}(*)$ & $\mathrm{s}(* *)$ & $\mathrm{s}(*)$ & ns & $\mathrm{ns}$ & ns & $\mathrm{s}(*)$ & $\mathrm{ns}$ & $\mathrm{s}(* *)$ & ns & $\mathrm{s}(*)$ \\
\hline
\end{tabular}




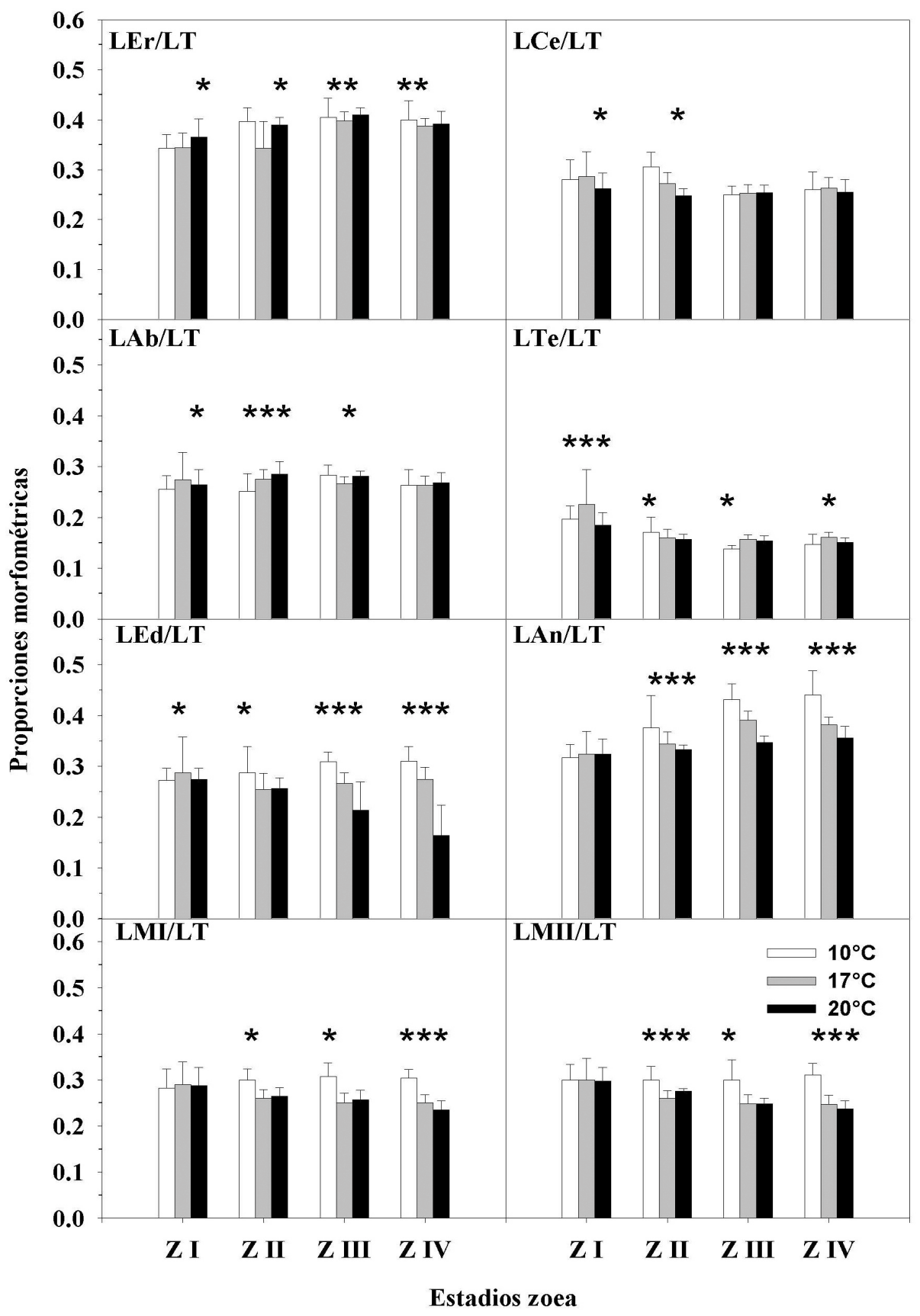

Figura 5. Proporciones morfométricas de la longitud estructural respecto de la longitud larval de la espina rostral (LEr/LT), cefalotórax (LCe/LT), abdomen (LAb/LT), telson (LTe/LT), espina dorsal (LEd/LT), antena (LAn/LT), primer maxilípedo (LMI/LT) y segundo maxilípedo (LMII/LT) en larvas zoeas cultivadas a 10, 17 y $20^{\circ}$ C. Las columnas representan valores promedios ( \pm desviación estándar $)$. (*) diferencias estadísticamente significativas $(\mathbf{p}<\mathbf{0 , 0 5})$.

Figure 5. Morphometrics proportions of the structural length versus the length of the rostral spine (LEr/LT), cephalothorax (LCe/LT), abdomen (LAb/LT), telson (LTe/LT), dorsal spine (LEd/LT), antenna (LAn/LT), first maxilliped (LMI/LT) and second maxilliped (LMII/LT) in zoeae larvae reared at 10, 17 and $20^{\circ} \mathrm{C}$. Columns represent average values $( \pm$ standard deviation). $(*)$ statistically significative differences $(\mathbf{p}<0.05)$. 
más baja, el desarrollo planctónico es más prolongado en el tiempo y por ello presentaría un potencial de dispersión significativamente mayor, con lo que se pudiesen establecer eventuales diferencias en variables poblacionales asociadas directamente con la dispersión larval, tales como el nivel de flujo génico, la tasa de intercambio genético entre distintas poblaciones (Scheltema, 1971; Palumbi, 1995), o el modelo de dinámica poblacional en poblaciones abiertas (Gaines \& Lafferty, 1995), dado en este caso por un gradiente latitudinal en la metapoblación de A. hassleri.

A. hassleri presentó, en todos los estadios de zoea, valores de longitud corporal significativamente mayores en los tratamientos a 20 y $17^{\circ} \mathrm{C}$ respecto de los cultivos a $10^{\circ} \mathrm{C}$ (Fig. 3, Tabla 3). En este sentido, Kinne (1970) postula que dentro de un grupo taxonómico frecuentemente los mayores tamaños larvales se han observado en los sectores más fríos del área de distribución, idea conocida como "Regla de tamaño de Bergman" (Anger, 2001). Para el caso de los crustáceos decápodos, esta tendencia ha sido observada en $C$. magister en el hemisferio norte (Shirley et al., 1987) y N. magellanica en el hemisferio sur (Wehrtmann \& Albornoz, 1998; Thatje \& Bacardit, 2000) donde las zoeas de mayor tamaño han sido registradas en las zonas de aguas más frías asociadas con las más altas latitudes dentro de sus respectivos rangos de distribución geográfica. Esta situación también ha sido observada en laboratorio, donde estudios acerca de la respuesta embrionaria a la temperatura de incubación en crustáceos decápodos ha dado como resultado que en los estadios finales de la embriogénesis los huevos de mayor volumen se han logrado con las menores temperaturas de cultivo, obteniendo en consecuencia larvas zoeas recién eclosionadas de tamaño significativamente mayor con las temperaturas de cultivo más bajas; tal es el caso de C. magister (Shirley et al., 1987) y del camarón Betaeus emarginatus Milne Edwards (Wehrtmann \& López, 2003). Por su parte, varios estudios en el ambiente natural avalan esta variación del tamaño en los embriones dado por una gradiente latitudinal en la distribución de las especies de crustáceos decápodos e.g. N. magellanica (Wehrtmann \& Kattner, 1998), Betaeus truncatus Dana (Lardies \& Wehrtmann, 2001), Pinnaxodes chilensis Milne Edwards (Lardies \& Castilla, 2001) y también en otros grupos de crustáceos tales como los isópodos Excirolana brazilienis Richardson (Cardoso \& Defeo, 2003) y E. hirsuticauda Menzies (Contreras \& Jaramillo, 2003).
Los tamaños larvales obtenidos en nuestros experimentos constituyen una excepción a la regla de tamaño de Bergman; asemejándose a las variaciones de tamaño observadas en larvas zoeas de $C$. magister por Sulkin \& McKeen (1996); quienes simulando en laboratorio distintas condiciones térmicas obtuvieron zoeas de mayor longitud en tratamientos de cultivo de mayor temperatura. No obstante, resulta necesario destacar que en ambos estudios se trabajó con larvas zoeas provenientes de huevos en avanzado estado de desarrollo embrionario, que fueron obtenidos de un mismo lugar y sometidos a un mismo régimen térmico de incubación. En este sentido, se sugiere que en ambos casos (Sulkin \& McKeen, 1996 y este trabajo), los resultados en términos de tamaño larval son la consecuencia de una respuesta de las larvas zoeas a una anomalía ambiental provocada por el cambio repentino de las condiciones térmicas durante la ontogenia temprana de estos animales, específicamente entre el final de la etapa embrionaria y el comienzo de la fase larval planctónica. Esto permite pensar que al someter larvas zoeas a distintos regímenes térmicos a partir de huevos provenientes de iguales condiciones ambientales se producen respuestas de crecimiento larval diferentes a las obtenidas en el ambiente natural. Por ello, es probable que la interpretación de los resultados bajo estas condiciones ambientales surja como respuesta a una anomalía ambiental exógena dentro del curso de las condiciones ambientales naturales. Al respecto, algunos autores (Anger, 2001; Wehrtmann \& Albornoz, 2003) argumentan que parte de la variabilidad en el desarrollo larval de crustáceos decápodos puede surgir como un artefacto de laboratorio, producto de que se le entregan condiciones ambientales controladas pero a su vez inapropiadas para el óptimo desarrollo larval. Es por ello que este aspecto es importante de ser tomado en cuenta en futuros experimentos de interpretación de resultados en laboratorio para tratar de explicar procesos naturales de la ontogenia larval.

No obstante, la longitud corporal de las larvas zoeas bajo las condiciones de manipulación descritas en este trabajo resultan aparentemente en un eventual artefacto de laboratorio; la respuesta en el incremento de tamaño de las espinas en función de la temperatura ambiental, es decir, una mayor longitud de las espinas del caparazón con menores temperaturas de cultivo se asemejan a las respuestas encontradas por Shirley et al. (1987) para las larvas de C. magister. Lo anterior, aparentemente refleja 
que la regulación del tamaño de las espinas por la temperatura ciertamente corresponde a un carácter conservativo para las larvas planctónicas de crustáceos decápodos, y que surge como una respuesta adaptativa al tiempo de permanencia en la columna de agua, aspecto que se discute con más detalle en los próximos párrafos, pero que sin duda requiere de mayor argumentación experimental.

Tanto el aumento de tamaño de las estructuras zoeales como el incremento de la longitud larval estuvo estrechamente relacionada con la temperatura de cultivo, aunque tal aumento de longitud no se manifestó en la misma proporción para todas las estructuras. En el presente estudio se observó que la espina dorsal (LEd), antena (LAn), primer y segundo maxilípedo (LMI y LMII) presentaron una relación de tamaño inversamente proporcional a la temperatura de cultivo (mayor tamaño a temperatura más baja), mientras que la espina rostral (LEr), cefalotórax (LCe), abdomen (LAb) y telson (LTe) presentaron tamaños directamente proporcionales a la temperatura de cultivo (mayores longitudes a temperaturas más altas) (Fig. 4). Por su parte, la proporción morfométrica de las estructuras larvales también presentó diferencias respecto de la temperatura de cultivo, es así como las variables LEd/LT, LAn/ LT, LMI/LT y LMII/LT registraron los mayores valores de proporción a $10^{\circ} \mathrm{C}$ a partir del estadio ZII y en la etapa ZIV para la variable LEr/LT (Fig. 5). Lo anterior indica que no todas las estructuras presentan el mismo patrón de crecimiento al estar sometidas a una misma temperatura. Al respecto, Shirley et al. (1987) encontraron que en el estadio ZI de $C$. magister proveniente de bajas temperaturas presentó espinas del caparazón de mayor tamaño, lo que interpretaron como el resultado de un mecanismo de crecimiento diferencial dado por un estímulo térmico denominado crecimiento alométrico termorregulado, el cual se encuentra condicionado por un proceso de plasticidad del desarrollo llamado modulación fenotípica, consistente en variaciones no específicas del fenotipo producidas por influencias ambientales provocando la expresión gradual de un programa de desarrollo sin que sea alterado el programa genético del individuo (Smith-Gill, 1983).

Sin considerar la longitud corporal total, el crecimiento estructural externo regulado por la temperatura permite que en el desarrollo zoeal de $A$. hassleri se pueda establecer un gradiente morfológico que va desde zoeas cultivadas a bajas temperaturas, provistas de espinas más largas, hasta zoeas cultivadas a mayor temperatura con apéndices corporales proporcionalmente más pequeñas. Al asociar el régimen térmico, la variabilidad morfométrica y el rango latitudinal de distribución de A. hassleri se visualiza la siguiente situación: en el límite norte de su distribución $\left(\mathrm{ca} .8^{\circ} \mathrm{N}\right)$, se esperaría encontrar zoeas con espinas proporcionalmente más pequeñas por efecto de la mayor temperatura del mar; mientras que, en el límite sur de su distribución $\left(\mathrm{ca} .40^{\circ} \mathrm{S}\right)$ producto de la menor temperatura del mar las larvas zoeas deberían estar provistas de espinas proporcionalmente más grandes.

En el contexto de la ecología larval, la variación morfológica gradual en el patrón de espinación por efecto directo de la temperatura e indirecto de la latitud, estaría relacionado con un modelo de adaptación apoyado en la idea del tiempo de permanencia de la larva zoea en el plancton. Al respecto, se ha señalado que las espinas de las larvas zoeas de crustáceos decápodos, así como también de otros organismos planctónicos, presenta fundamentalmente dos funciones: como potencial mecanismo de defensa contra predadores planctónicos, así como también para la regulación de las migraciones verticales, estabilización del organismo en la columna de agua o regulación de su velocidad de hundimiento (Cowden et al., 1984; Pennintong \& Chia, 1984; Sulkin, 1984; Shirley et al., 1987; Morgan, 1987, 1989, 1990; Morgan \& Christy, 1997).

De acuerdo con la Figura 2a, una larva zoea de A. hassleri que se desarrolla en un ambiente a $10^{\circ} \mathrm{C}$ (altas latitudes), permanecerá en la columna de agua un tiempo superior al doble de lo que permanecerá una larva que se encuentre a $20^{\circ} \mathrm{C}$ (bajas latitudes). Esto implica que la larva de aguas más frías presentará un mayor grado de exposición a depredadores planctónicos aumentando la probabilidad de encuentro y captura por parte de un organismo planctívoro (Levin \& Bridges, 1995). Esta desventaja sería compensada en larvas de aguas más frías por espinas, antenas y maxilípedos de mayor longitud como mecanismo de defensa contra la predación y también como estrategia de ahorro energético en la mantención de la larva en la columna de agua, puesto que al tener espinas de mayor tamaño aumenta la flotabilidad, y al poseer maxilípedos de mayor tamaño mejoraría la capacidad natatoria y, en consecuencia, aumenta su autonomía de movimiento. 


\section{AGRADECIMIENTOS}

Este trabajo fue parcialmente financiado por los proyectos DID-E-97-03 y DID-S-98-17 de la Dirección de Investigación y Desarrollo de la Universidad Austral de Chile. Durante la preparación del manuscrito, el primer autor fue apoyado por la Escuela de Graduados de la Universidad Austral de Chile y el Programa de Becas Doctorales MECESUP AUS0111. Agradecemos los comentarios del Sr. Luis Albornoz y de revisores anónimos que ayudaron a mejorar el contenido de este trabajo.

\section{REFERENCIAS}

Anger, K. 1983. Temperature and the larval development of Hyas araneus L. (Decapoda: Majidae); extrapolation of laboratory data to fields conditions. J. Exp. Mar. Biol. Ecol., 69: 203-215.

Anger, K. 2001. The biology of decapod crustacean larvae. Crustacean Issues, 14. AA Balkema, Lisse, $405 \mathrm{pp}$.

Anger, K., R.R. Dawirs, V. Anger, J.W. Goy \& J.D. Costlow. 1981. Starvation resistance in first stage zoeae of brachyuran crabs in relation to temperature. J. Crust. Biol., 1: 518-525.

Anger, K., S. Thatje, G. Lovrich \& J. Calcagno. 2003. Larval and early juvenile development of Paralomis granulosa reared at different temperatures: tolerance of cold and food limitation in a lithodid crab from high latitudes. Mar. Ecol. Progr. Ser., 253: 243-251.

Báez, P. 1997. Key to the families of decapod crustacean larvae collected off northern Chile during an El Niño event. Invest. Mar., Valparaíso, 25: $167-176$.

Cardoso, R.S. \& O. Defeo. 2003. Geographical patterns in reproductive biology of the PanAmerican sandy beach isopod Exirolana braziliensis. Mar. Biol., 143: 573-581.

Carrasco, R.A. \& J.H. Zamorano. 2000. Fecundidad y periodo reproductivo en poblaciones simpátricas de Acanthocyclus gayi Milne Edwards \& Lucas, 1844 y A. hassleri Rathbun, 1898 (Decapoda: Atelecyclidae) en el intermareal rocoso de Pichicullín (X Región, Chile). Rev. Biol. Mar. Oceanogr., 35(1): 103-110.
Castilla, J.C., C. Luxoro \& S.A. Navarrete. 1989. Galleries of the crabs Acanthocyclus under intertidal mussel beds: their effects on the use of primary substrate. Rev. Chil. Hist. Nat., 62: 199204.

Clancy, M. \& J.S. Cobb. 1997. Effect of wind and tidal advection on distribution patterns of rock crab Cancer irroratus megalopae in Block Island Sound, Rhode Island. Mar. Ecol. Progr. Ser., 152: 217-225.

Contreras, H. \& E. Jaramillo. 2003. Geographical variation in natural history of the sandy beach isopod Exirolana hirsuticauda Menzies (Cirolanidae) on the Chilean coast. Est. Coast. Shelf Sci., 58: 117-126.

Cowden, C., C.M. Young \& F.S. Chia. 1984. Differential predation on marine invertebrate larvae by two benthic predators. Mar. Ecol. Progr. Ser., 14: $145-149$.

Epifanio, C.E. 1987. The role of tidal fronts in maintaining patches of brachyuran zoeae in estuarine waters. J. Crust. Biol., 7: 513-517.

Gaines, S.D. \& K.D. Lafferty. 1995. Modeling the dynamics of marine species: The importance of incorporating larval dispersal. En: L.R. McEdward (ed.). Ecology of marine invertebrate larvae. CRC Press, New York, pp. 389-411.

Garrison, L.P. 1999. Vertical migration behavior and larval transport in brachyuran crabs. Mar. Ecol. Progr. Ser., 176: 103-113.

Gebauer, P., K. Paschke \& K. Anger. 2003. Delayed metamorphosis in Decapod crustaceans: evidence and consequences. Rev. Chil. Hist. Nat., 76: 169175 .

Gill, J.L. 1978. Design and analysis of experiments in the animal and medical sciences. Vol. I. Iowa State University Press, Iowa, 409 pp.

Ismael, D., K. Anger \& G.S. Moreira. 1997. Influence of temperature on larval survival, development, and respiration in Chasmagnathus granulata (Crustacea, Decapoda). Helgoländer Meeresunters., 51: 463-475.

Johns, D.M. 1981. Physiological studies in Cancer irroratus larvae. I. Effects of temperature and salinity on survival, development rate and size. Mar. Ecol. Progr. Ser., 5: 75-83. 
Kinne, O. 1970. Temperature. Invertebrates. En: O. Kinne (ed.). Marine ecology. Wiley \& Sons, Chichester, pp. 407-415.

Lardies, M.A. \& J.C. Castilla. 2001. Latitudinal variation in the reproductive biology of the commensal crab Pinnaxodes chilensis (Decapoda: Pinnotheridae) along the Chilean coast. Mar. Biol., 139: 1125-1133.

Lardies, M.A. \& I.S. Wehrtmann. 2001. Latitudinal variation in the reproductive biology of Betaeus truncatus (Decapoda: Alpheidae) along the Chilean coast. Ophelia, 55: 55-67.

Laughlin, R.B. \& J.M. Neff. 1981. Ontogeny of respiratory and growth responses of larval mud crabs Rhithropanopeus harrisii exposed to different temperatures, salinities and naphthalene concentrations. Mar. Ecol. Progr. Ser., 5: 319-332.

Levin, L.A \& T.S. Bridges. 1995. Pattern and diversity in reproduction and development. En: L. McEdwards (ed.). Ecology in marine invertebrate larvae. CRC Press, New York, pp. 1-48.

Minagawa, M. 1990. Influence of temperature on survival, feeding and development of larvae of the frog crab, Ranina ranina (Crustacea, Decapoda, Raninidae). Nippon Suisan Gakkaishi, 56: 755-760.

Morgan, S.G. 1987. Morphological and behavioral antipredatory adaptations of decapod zoeae. Oecologia, 73: 393-400.

Morgan, S.G. 1989. Adaptative significance of spination in estuarine crab zoeae. Ecology, 70: 464482.

Morgan, S.G. 1990. Impact of planktivorous fishes on dispersal, hatching, and morphology of estuarine crab larvae. Ecology, 71: 1639-1652.

Morgan, S.G. 1992. Predation by planktonic and benthic invertebrates on larvae of estuarine crabs. J. Exp. Mar. Biol. Ecol., 163: 91-110.

Morgan, S.V. \& J.H. Christy. 1997. Planktivorous fishes as selective agents for reproductive synchrony. J. Exp. Mar. Biol. Ecol., 209: 89-101.

Natunewicz, C.C. \& C.E. Epifanio. 2001. Spatial and temporal scales of patches of crab larvae in coastal waters. Mar. Ecol. Progr. Ser., 212: 217-222.
Natunewicz, C.C., C.E. Epifanio \& R.W. Garvine. 2001. Transport of crab larval patches in the coastal ocean. Mar. Ecol. Progr. Ser., 222: 143-154.

Navarrete, S. \& J.C. Castilla. 1988. Foraging activities of Chilean intertidal crabs Acanthocyclus gayi Milne-Edwards et Lucas and A. hassleri Rathbun. J. Exp. Mar. Biol. Ecol., 118: 115-136.

Palumbi, S.R. 1995. Using genetics as an indirect estimator of larval dispersal. En: L. McEdwards (ed.). Ecology in marine invertebrate larvae. CRC Press, New York, pp. 369-388.

Pechenick, J.A. 1987. Enviromental influences on larval survival and development. En: A.C. Giese, J.S. Pearse $\&$ V.B. Pearse (eds.). Reproduction of marine invertebrates Vol. 9. Blackwell Scientific Publications and Boxwood Press, California, pp. 551-608.

Pennington, J.T. \& F.S. Chia. 1984. Morphological and behavioral defenses of trochophore larvae of Sabellaria cementarium (Polychaeta) against four planktonic predators. Biol. Bull., 167: 168-175.

Retamal, M.A. 1981. Catálogo ilustrado de los crustáceos decápodos de Chile. Gayana Zool., 44: 7 110 .

Sastry, A.N. 1983. Pelagic larval ecology and development. En: D.E. Bliss (ed.). The biology of crustacea. Vol. 7. Behavior and Ecology. Academic Press, New York, pp. 214-282.

Scheltema, R.S. 1971. Larval dispersal as a means of genetic exchange between geographically separated populations of shallow-water benthic marine gastropods. Biol. Bull., 140: 284-322.

Sepúlveda, M.A. 1996. Descripción del desarrollo larval de Acanthocyclus hassleri Rathbun, 1898 (Crustacea, Decapoda, Atelecyclidae) en condiciones de laboratorio. Tesis de Biología Marina, Universidad Austral de Chile, Valdivia, 40 pp.

Shirley, S.M., T.C. Shirley \& S.D. Rice. 1987. Latitudinal variation in the dungeness crab, Cancer magister: zoeal morphology explained by incubation temperature. Mar. Biol., 95: 371-376.

Smith-Gill, S.J. 1983. Developmental plasticity: Developmental conversion versus phenotypic modulation. Amer. Zool., 23: 47-55.

Sokal, R.R. \& F.J. Rohlf. 1995. Biometry. The principles and practice of statistics in biological research. Freeman, New York, 887 pp. 
Sulkin, S.D. 1984. Behavioral basis of depth regulation in the larvae of brachyuran crabs. Mar. Ecol. Progr. Ser., 15: 181-205.

Sulkin, S.D. \& G.L. McKeen. 1996. Larval development of the crab Cancer magister in temperature regimes simulating outer-coast and inland-water habitats. Mar. Biol., 127: 235-240.

Sulkin, S.D., E. Mojica \& G.L. McKeen. 1996. Elevated summer temperature effects on megalopa and early juvenile development in the dungeness crab, Cancer magister. Can. J. Fish. Aquat. Sci., 53: 2076-2079.

Thatjte, S. \& R. Bacardit. 2000. Morphological variability in larval stages of Nauticaris magellanica (A. Milne Edwards, 1891) (Decapoda: Caridea: Hippolytidae) from South American waters. Bull. Mar. Sci., 66: 375-398.

Villamar, D.F. \& G.J. Brusca. 1988. Variation in the larval development of Crangon nigricauda (Decapoda: Caridea), with notes on larval morphology and behavior. J. Crust. Biol., 8: 410419.

Wehrtmann, I.S. \& L.A. Albornoz. 1998. Larval development of Nauticaris magellanica (A. Milne Edwards, 1891) (Decapoda: Caridea: Hippolyti- dae), reared under laboratory conditions. Bull. Mar. Sci., 62: 45-72.

Wehrtmann, I.S. \& L.A. Albornoz. 2003. Larvae of Nauticaris magellanica (Decapoda: Caridea: Hippolytidae) reared in the laboratory differ morphologically from those in nature. J. Mar. Biol. Ass. U.K., 83: 949-957.

Wehrtmann, I.S. \& G. Kattner. 1998. Changes in volume, biomass and fatty acids of developing eggs in Nauticaris magellanica (Decapoda: Caridea): a latitudinal comparison. J. Crust. Biol., 18: 413-422.

Wehrtmann, I.S. \& G.A. López. 2003. Effects of temperature on the embryionic development and hatchling size of Betaeus emarginatus (Decapoda: Caridea: Alpheidae). J. Nat. Hist., 37: 2165-2178.

Williamson, D.I. 1982. Larval morphology and diversity. En: D.E. Bliss (ed.) The biology of Crustacea, Vol. 2. Academic Press, New York, pp. 43110.

Zagal, C., C. Hermosilla \& A. Riedemann. 2001. Guía de invertebrados marinos del litoral valdiviano. Universidad Austral de Chile, Valdivia, $215 \mathrm{pp}$. 